\title{
Machine-Learnt Turbulence Closures for Low Pressure Turbines with Unsteady Inflow Conditions
}

\author{
H.D. Akolekar; R.D. Sandberg, \\ N. Hutchins \\ Department of Mechanical Engineering \\ University of Melbourne \\ Parkville, VIC 3010, Australia \\ Email: hakolekar@student.unimelb.edu.au
}

\author{
V. Michelassi \\ Baker Hughes, a GE Company \\ Florence 50127, Italy \\ vittorio.michelassi@bhge.com
}

\author{
G. Laskowski \\ General Electric Aviation \\ Lynn, MA 01905, USA
}

\begin{abstract}
The design of low-pressure turbines (LPT) must account for the losses generated by the unsteady interaction with the upstream blade row. The estimation of such unsteady wake induced losses requires the accurate prediction of the incoming wake dynamics and decay. Existing linear turbulence closures (stressstrain relationships), however, do not offer an accurate prediction of the wake mixing. Therefore, machine-learnt, non-linear turbulence closures (models) have been developed for LPT flows with unsteady inflow conditions using a zonal based model development approach, with an aim to enhance the wake mixing prediction for unsteady Reynolds-averaged Navier-Stokes calculations. High-fidelity time-averaged and phase-lock averaged data at a realistic isentropic Reynolds number and two reduced frequencies, i.e. with discrete incoming wakes and with wake 'fogging', have been used as reference data for machine learning algorithm gene expression programming to develop models. Models developed via phase-lock averaged data were able to capture the effect of certain prominent physical phenomena in LPTs such as thinar separation and wake-wake interactions, whereas models based on the time-averaged data could not. Correlations with the flow physics lead to a set of models that can effectively enhance the wake mixing prediction across the entire LPT domain for both cases. Based on a newly developed error metric, the developed models have reduced the error over the Boussinesq approximation on average by $45 \%$, a priori; thereby enhaneing the wake mixing prediction. This study thus aids blade designers in selecting the non-linear closures capable of mimicking the physical mechanisms responsible for loss generation.
\end{abstract}

\section{Introduction}

Low-pressure turbines (LPT) experience a challenging range in Reynolds numbers from 50,000 to 250,000 [1]. Different physical phenomena occur throughout this range of Reynolds numbers. The boundary layer on the suction side, which is largely laminar in nature, becomes prone to flow separation at moderately-low Reynolds numbers in regions of local-adverse pressure gradients. The selection of LPT design parameters, like the vane and blade count, the revolution speed, and the axial flow velocity $\left(V_{x}\right)$, concurrently determine the values of both the flow coefficient $(\Phi)$, and the reduced frequency $\left(F_{\text {red }}\right)$. These two parameters control the dynamics of the periodic unsteady incoming wakes from the previous blade-rows [2], which are the dominant

\footnotetext{
*Address all correspondence to this author.
}

source of unsteady losses in LPTs. These wakes interact with and perturb the suction side boundary layer and affect the laminar separation onset, size of the separation bubble and its turbulent reattachment. Moreover, these wakes also induce other wake-wake interactions in the flow-field. A number of experimental studies [1,3-6], and high fidelity simulations [2,7-10] have been conducted to investigate the nature and influence of these unsteady phenomenon on the performance of LPTs. All of these phenomena affect the wake mixing in LPTs which in turn influences the losses generated and thus the net efficiency. Praisner et al. [11] suggested that the losses due to wake mixing can contribute up to 1.5 percent of lost efficiency in LPTs.

Much of the design process for LPTs is, however, conducted using Reynolds-averaged Navier-Stokes (RANS) based turbulence models. Accurate prediction of the wake mixing and other complex phenomena in LPTs is still a challenge for existing RANS models as shown by a number of studies [12-14]; and there is plenty of scope for improvement in these models. One of the reasons that RANS closures fail at predicting the turbulent wake mixing with the required degree of accuracy is the use of the Boussinesq approximation [15] for the stress-strain relationship. There are numerous approaches to address the shortcomings in this relationship, however, the most computationally efficient approach to address the shortcomings of the Boussinesq approximation are explicit algebraic Reynolds stress models (EARSM) [16]. A number of EARSMs have been developed for turbulent flows $[17,18]$ using analytical approaches; however, this study uses a machine-learning based approach for non-linear EARSM development to take full advantages of a set of high fidelity simulations.

Data-driven turbulence modeling is an upcoming field with great potential that has been applied to a multitude of cases using a number of methods [19]. Duraisamy et al. [20] used nondimensional flow variables to modify the Spalart Allmaras model using neural networks and inverse modeling. These models were used by Singh et al. [21] which showed good generalization capabilities for a class of flows around airfoils. Ling et al. [22] showed the importance of embedding tensorial invariance within machine learning approaches and used deep neural networks to improve Reynolds stress predictions for ducts. Wang et. al [23] proposed a data-driven approach to improve discrepancies in RANS modeled Reynolds stresses for two geometries: periodic hills and a square duct. However, even though they improved the Reynolds stress prediction, they could not translate this improvement to mean flow fields. This issue arose due to ill-conditioned 
models. Wu et al. [24] proposed a method to overcome the illconditioning issue, and the models that were thus developed not only brought improvement in the mean flow-field but also over different test cases. Milani et al. [25] used random forests to improve heat-flux modeling for film cooling applications.

Ferreira [26] introduced gene expression programming (GEP) which was a new adaptive algorithm for solving regression based problems. Weatheritt \& Sandberg [27] developed GEP for turbulence modeling, which creates new EARSM closures imposing Galilean invariance. GEP for turbulence modeling produces symbolic expressions which are physically interpretable. These expressions can be correlated with certain physical phenomena, unlike neural networks, wherein the analytical form of the model remains hidden within the network. GEP is relatively inexpensive as compared to other machine learning techniques used for previous turbulence modeling studies as the models generated via this method have an added advantage that they do not require any supporting machine-learning or high fidelity databases for their implementation into computational fluid dynamics codes. The derived closures can be directly plugged into RANS codes. The algorithm not only improved the Reynolds stress prediction, but also improved the mean flow features for a few canonical cases [28]. GEP was applied for the first time on a turbomachinery component when an a priori study was conducted for high pressure turbines [29]. The EARSMs developed brought about a $32 \%$ reduction in error over the baseline case in an a priori sense. However, these models were not implemented into RANS calculations. Akolekar et al. [30] not only developed a family of EARSMs for LPT flows with steady inflow conditions, but also implemented them into unsteady-RANS (URANS) calculations and tested them across two topologically different flows in order to enhance wake mixing prediction. The models not only offered significant improvements in terms of secondary statistics such as turbulent kinetic energy (TKE) production; wherein they reduced error in peak TKE production relative to direct numerical simulations (DNS) from $70 \%$ (baseline case with Boussinesq approximation) to up to $12 \%$, but also improved mean flow features such as velocity, loss profiles and wake maturity, etc. These models demonstrated a good degree of robustness across the two cases tested. A similar study was conducted by Sandberg et al. [31] for a trailing edge (TE) slot using GEP to enhance heat flux modeling across two lip thickness ratios.

This study extends the analysis conducted by Akolekar et al. [30] to LPTs with unsteady inflow conditions by developing a framework, that, for the first time will produce EARSMs which enhance the wake mixing prediction of these cases. The focus of this work is the midspan section of the LPT, which is responsible for at least $60 \%$ of the total loss in an LPT [7]. Large eddy simulation (LES) data for two cases with an isentropic exit Reynolds number of 100,000 and reduced frequencies of 0.624 and 1.866 have been used as reference data for model generation. Using the phase-lock averaged and time-averaged data that is available, three different approaches have been used to develop a large family of models. The detailed assessment and performance in the $a$ priori sense of the models that are developed from each of these approaches forms the majority of this study. An extensive discussion about the correlation of the model coefficients with physical phenomena has also been addressed. After a robustness study of these models across the different phases and cases, a few models have been selected for conducting future URANS calculations. It is expected that this study offers blade designers an additional insight into the kinds of linear and non-linear stress-strain relationships (EARSMs) that are required to improve the accuracy of existing URANS modeling techniques for LPTs with unsteady inflow conditions.

\section{EARSMs for Turbulence Models}

The Boussinesq approximation, which is one of the major sources of error in RANS modeling, can be broken up into the sum of isotropic and anisotropic contributions

$$
\boldsymbol{\tau}_{i j}=\underbrace{\frac{2}{3} \rho k \boldsymbol{\delta}_{i j}}_{\text {isotropic }}-\underbrace{2 \mu_{t}\left(\boldsymbol{S}_{i j}-\frac{1}{3} \frac{\partial u_{k}}{\partial x_{k}} \boldsymbol{\delta}_{i j}\right)}_{\text {anisotropic }} .
$$

Of the many of ways to address the deficiencies of the Boussinesq stress-strain relation, EARSMs are an effective and computationally efficient approach. Beginning from the weak equilibrium hypothesis [32], a class of turbulence closures, known as EARSMs [16] were developed to express the anisotropy tensor $\boldsymbol{a}_{i j}$ as a function of a tensor basis $\boldsymbol{V}_{i j}^{m}$ and scalar invariants $I_{k}$. These models add non-linear terms to the stress-strain relationship and their calculation incurs little additional computational expense. Equation 2 defines $\boldsymbol{a}_{i j}$, which is normalized by $2 \rho k$, for both the Boussinesq approximation and EARSMs

$$
\begin{aligned}
\boldsymbol{a}_{i j} & \equiv \frac{\boldsymbol{\tau}_{i j}}{2 \rho k}-\frac{1}{3} \boldsymbol{\delta}_{i j}, \\
\boldsymbol{a}_{i j}^{B} & =-\frac{v_{t}}{k} \boldsymbol{S}_{i j}^{\prime} \quad(\text { Boussinesq }), \\
\boldsymbol{a}_{i j}^{E A R S M} & =-\frac{v_{t}}{k} \boldsymbol{S}_{i j}^{\prime}+f\left(\boldsymbol{V}_{i j}^{1}, \boldsymbol{V}_{i j}^{2}, \boldsymbol{V}_{i j}^{3}, \ldots, I_{1}, I_{2}, \ldots\right) . \\
\boldsymbol{a}_{i j}^{E A R S M} & =-\frac{v_{t}}{k} \boldsymbol{S}_{i j}^{\prime}+\sum_{m=1}^{4}\left(\zeta_{m} \boldsymbol{V}_{i j}^{m}\right)
\end{aligned}
$$

Note that the $-\frac{v_{t}}{k} \boldsymbol{S}_{i j}^{\prime}$ term is maintained in the anisotropy relation in Eq. $2 d$ for the EARSM model in order to maintain numerical stability when these models are implemented into (U)RANS solvers. The tensor basis and set of scalar invariants, used to define $\boldsymbol{a}_{i j}^{E A R S M}$, are functions of the non-dimensional strain and rotation rate tensors, denoted by $\boldsymbol{s}_{i j}=\tau \boldsymbol{S}_{i j}^{\prime}$ and $w_{i j}=\tau \boldsymbol{\Omega}_{i j}$; where $\tau$ is the turbulent time scale denoted by $1 / \omega$. For the flow scenario considered in this work, only the tensor basis functions up to the second order have been considered. The selected tensor basis functions and invariants are:

$$
\begin{aligned}
& \boldsymbol{V}_{i j}^{1}=\boldsymbol{s}_{i j}, \boldsymbol{V}_{i j}^{2}=\boldsymbol{s}_{i k} \boldsymbol{w}_{k j}-\boldsymbol{w}_{i k} \boldsymbol{s}_{k j} \\
& \boldsymbol{V}_{i j}^{3}=\boldsymbol{s}_{i k} \boldsymbol{s}_{k j}-\frac{1}{3} \boldsymbol{\delta}_{i j} \boldsymbol{s}_{m n} \boldsymbol{s}_{n m} \\
& \boldsymbol{V}_{i j}^{4}=\boldsymbol{w}_{i k} \boldsymbol{w}_{k j}-\frac{1}{3} \boldsymbol{\delta}_{i j} \boldsymbol{w}_{m n} \boldsymbol{w}_{n m} \\
& I_{1}=\boldsymbol{s}_{m n} \boldsymbol{s}_{n m}, I_{2}=\boldsymbol{w}_{m n} \boldsymbol{w}_{n m}
\end{aligned}
$$

The data used for model development in this study has been spanwise averaged. This averaging sets the shear components (other 
than $\boldsymbol{\tau}_{x y}$ ) of the Reynolds stress tensor to zero. However, the normal component of the Reynolds stress in the third direction $\left(\tau_{z z}\right)$ does continue to exist. $\tau_{z z}$ is the normal stress component along the spanwise direction that arises from the isotropic component of the Boussinesq approximation and it contributes to the TKE. For EARSMs developed for statistically 2D flows in the past [18] only the first three tensor basis functions from Eq. 3 have been used. However, in this study the $4^{\text {th }}$ tensor basis function (which is the last quadratic tensor basis function) has also been used as it is non-zero due to the presence of $\tau_{z z}$. Other tensor basis functions have not been used, as it has been found that the contribution of these higher order tensor basis functions is negligible to the reduction of mean square error.

\section{LES Flow Configuration}

Table 1: List of LES configurations.

\begin{tabular}{|c|c|c|c|c|c|c|}
\hline Case & \# Bars & $\begin{array}{c}\text { Blade } \\
\text { pitch }\end{array}$ & $\begin{array}{c}\text { Bar } \\
\text { pitch }\end{array}$ & $\begin{array}{c}\text { Bar } \\
\text { speed }\end{array}$ & $\Phi$ & $\mathbf{F}_{\text {red }}$ \\
\hline 1B2U & 1 & 0.799 & 0.799 & 0.820 & 0.850 & 0.624 \\
\hline 2B3U & 2 & 0.799 & 0.400 & 1.230 & 0.569 & 1.866 \\
\hline
\end{tabular}

A number of highly-resolved LES were carried out by Michelassi et al. [2] for the mid-span section of a T106A LPT blade, which has a pitch to chord ratio of 0.799. It is these datasets that have been used for the EARSM development outlined in this work. It has been demonstrated how it is possible to run LES [2] with a minimum loss in accuracy with respect to DNS [7], which in turn were shown to reproduce experimental results [33]. As the EARSM development process outlined in the methodology section does not rely on the exact dissipation rate from high-fidelity simulations, highly resolved and DNSvalidated LES databases can be used for developing EARSMs. The reference isentropic exit Reynolds number $\left(R e_{2 i s}\right)$ is 100,000
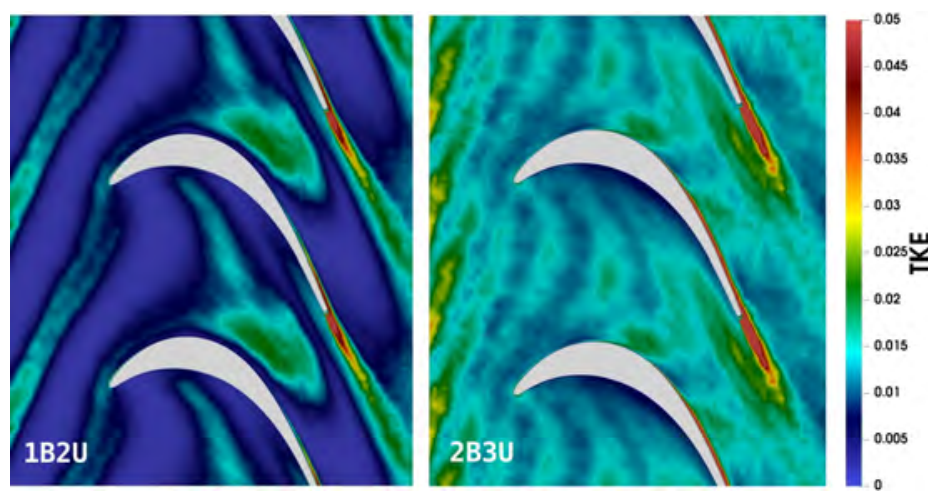

Fig. 1: Phase-lock averaged TKE snapshots for the (a) 1B2U and (b) 2B3U cases.

and the flow coefficient and reduced frequencies have been varied over certain ranges in order to offer a broad spectrum of qualitative information for blade designers. In order to develop un- steady inflow for the LPT, moving cylindrical bars (with a nondimensional speed of 0.41 ) were used $70 \%$ real chord upstream of the blade leading edge (LE). For the purpose of model development, two cases have been selected that show different flow features which are abbreviated as 1B2U and 2B3U. Table 1 depicts the flow configuration of these two cases. The blade and bar pitch are normalized with respect to the real chord. In a linear cascade with moving upstream bars the reduced frequency and the flow coefficient are defined as:

$$
F_{\text {red }}=\frac{U}{P_{b a r}} \frac{C}{V_{2}}, \quad \Phi=\frac{V_{x}}{U}
$$

Each case is referred to by its bar count and speed (i.e. 1B2U corresponds to 1 bar per blade pitch with a nominal bar speed of $0.82)$. An effective way to show how the combination of $F_{\text {red }}$ and $\Phi$ affects the incoming wake dynamics is to plot phase-lock averaged snapshots of the TKE, as this traces the path of the wake to the blade LE, into the passage and to the blade TE. Figure 1 depicts the TKE for phase-lock averaged flow fields of the 1B2U and $2 \mathrm{~B} 3 \mathrm{U}$ cases. One stark difference between the two cases is that discrete incoming wakes can be identified in the $1 \mathrm{~B} 2 \mathrm{U}$ case whereas in the 2B3U case, the wakes seem to merge with one another; also known as 'fogging'. The discrete unsteadiness that is exhibited by the 1B2U case seems to fade away as the bar speed and bar pitch are increased (2B3U), which leads to more pronounced wake-wake interaction at the inlet. The 1B2U case was chosen for model development as present turbines typically operate at $F_{\text {red }}<1$. The $2 \mathrm{~B} 3 \mathrm{U}$ case, as a limiting condition, represents a case of plain free-stream turbulence, as the discrete wakes start to merge and lose their discreteness. In order to receive insights for the EARSM development process across a wide spectrum of flow conditions, both of these cases have been selected. The EARSM development uses phase-lock averaged data sets; at 20 and 17 equispaced phase angles for the 1B2U and 2B3U cases, respectively, over one incoming wake passing period $(T)$. There are two ways of identifying particular phase-lock averaged flow fields; one of them simply refers to it by the phase number (i.e. phase 5 as p5); the other represents the data across one time period $(T)$. A simple conversion can be made between the time $(t)$ and the phase number as follows: $t / T=p / N$, where $\mathrm{N}$ is the total number of phases and $\mathrm{N}=20 \& \mathrm{~N}=17$ for the $1 \mathrm{~B} 2 \mathrm{U}$ and 2B3U cases respectively. The next section outlines the methodology for EARSM development.

\section{Machine Learning Methodology}

The EARSM development for LPTs with unsteady inflow conditions is carried out with GEP $[26,27]$ and this section describes the various aspects of the process.

GEP Algorithm Each of the phase-lock averaged datasets, for both the 1B2U and 2B3U cases, has been used as reference data to create EARSMs. The EARSM development approach is outlined in Fig. 2. As the focus of this work is to improve the wake mixing prediction, the Reynolds stresses (or indirectly the anisotropy field: $\boldsymbol{a}_{i j}^{L E S}$, calculated from Eq. 2a), velocity, temperature, TKE and density from a wake region are extracted from the LES and fed into the GEP algorithm. These data are also used to solve the $\overline{v_{2}}$ and $\omega$ equations of the $k \overline{v_{2}} \omega$ turbulence model [34] using the so-called anisotropy-based 'frozen' 


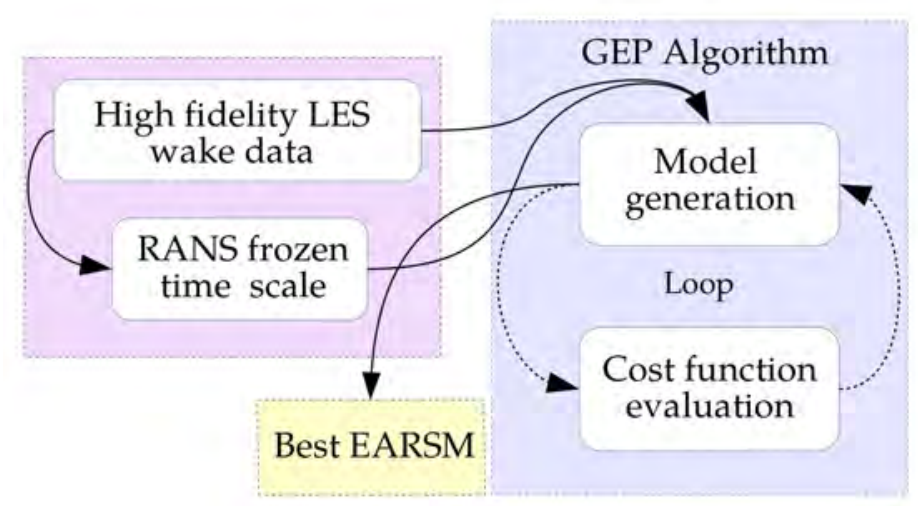

Fig. 2: The anisotropy-based 'frozen' approach with the GEP algorithm.

approach [35]. The $k \overline{v_{2}} \omega$ model has been used as in our numerical setup it was found to offer the best prediction of the boundary layer and wake losses as compared to other turbulence models for LPTs [30]. The above approach allows the extraction of a turbulence time scale $(1 / \omega)$ to normalize the tensor basis functions in Eq. 3. Theoretically, it is possible to extract a dissipation $(\omega)$ value from LES data; however, it has been found that this value does not in any way resemble the $\omega$ definition used in a (U)RANS context. Therefore, the 'frozen' approach is used in order to extract a turbulence time scale which is suitable for (U)RANS calculations. The strain-rate and rotation-rate tensors are calculated using LES data and are normalized with the RANS turbulence time scale. Using these normalized tensor basis functions, the GEP algorithm creates a family of models which are then evaluated against a cost function.

Cost Function GEP, a regression based algorithm, aims to minimize the sum of the difference between the individual components of the anisotropy tensors, i.e. the anisotropy tensor that is created from the EARSMs $\left(\boldsymbol{a}_{i j}^{E A R S M}\right)$ and the one from the LES flow field $\left(\boldsymbol{a}_{i j}^{L E S}\right)$, as

$$
J\left(\boldsymbol{a}_{i j}^{B / E A R S M}\right)=\frac{1}{M} \sum_{n=1}^{M} \sum_{i=1}^{3} \sum_{j \leq i}\left(\left|\boldsymbol{a}_{i j}^{L E S}-\boldsymbol{a}_{i j}^{B / E A R S M}\right|\right)^{2},
$$

where $M$ is the total number of points in the training region. The GEP algorithm attempts to find suitable values of $\zeta_{m}$ in Eq. 2 d. As this is an iterative process, the evolution of models keeps on going for a fixed number of iterations or until the cost function falls below a certain threshold. The GEP algorithm then returns an anisotropy tensor $\left(\boldsymbol{a}_{i j}^{E A R S M}\right)$, which best fits the $\boldsymbol{a}_{i j}^{L E S}$ tensor. Regression for the anisotropy tensor is sought, as it has been shown that improvements in the RANS wake mixing prediction can be brought about by a better representation of the anisotropy tensor [30]. The wake mixing process is governed by turbulent shear stresses. Ultimately, turbulence anisotropy is related to the differences between the turbulent shear stress components that control the production rate of turbulence. Therefore, the anisotropy tensor, i.e. a direct measure of the local turbulence anisotropy, is a good measure of the ability of a turbulence model to reproduce the fundamental physics that drives the diffusion process. The anisotropy tensor also appears in the form of the Reynolds stress tensor in the Navier-Stokes momentum equation and in the production terms of the TKE and $\omega$ transport

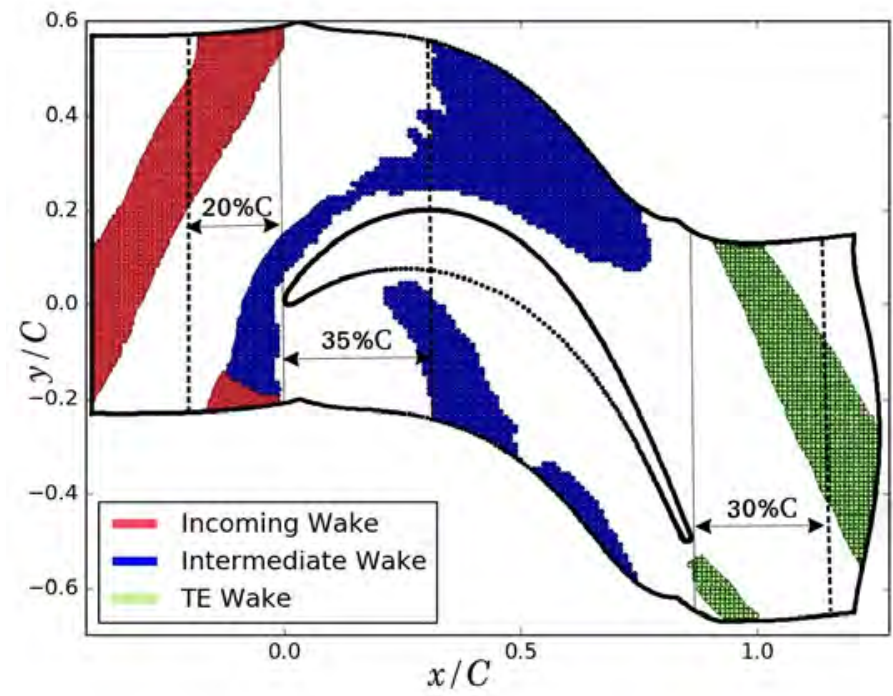

Fig. 3: Training regions for EARSM development for phase 1 of the 1B2U case. Also shown are some measurement locations.

equations. If the modeling for this term is improved, there will be better prediction of the wake diffusion and TKE production, which will lead to enhanced wake mixing.

Since the GEP is a non-deterministic algorithm, it depends on a random number seed. If this seed value is changed, every different run of the GEP produces a model with slightly different coefficients, while achieving comparable values of the cost function. In order to account for this slight variation, every model presented in this work is the result of running the GEP algorithm 25 times on that particular case and training region. The coefficients from those 25 runs of each of the tensor basis functions are averaged and presented as the ensemble-averaged model. The ensemble-averaged model ensures that the models do not overfit the data set and also ensure that any noise that is created by the individual models is removed.

Training Regions for Zonal Model Development It was found by Akolekar et al. [30] that selecting the appropriate data or training region to improve the wake mixing is particularly important. They showed that it is necessary to select the data in the wake region (which can be demarcated from the surrounding flow by values of TKE above a certain threshold), rather than a region which encompasses the wake and the area between the wakes (wake passage). However, in their study only the wake coming off the blade TE was considered for model development. In this study, models also need to be developed for the wakes which are incident on the blade LE and that are transported through the blade passage, in order to enhance the overall wake mixing in the LPT domain. In order to effectively develop EARSMs for these wakes, the magnitudes of the tensor basis functions and scalar invariants have to be assessed in the various wake regions. They are found to differ, broadly with the region in the flow-field. Figure 3 shows three different kinds of wake regions (for phase 1 of the $1 \mathrm{~B} 2 \mathrm{U}$ case), which are each dominated by different physical phenomena. The wakes which are incoming from the previous blade row (incoming wakes) tend to be dominated by turbulent diffusion whereas the wakes in the blade passage (intermediate wakes) undergo dilation-distortion and interact with the suction and pressure side boundary layers. 


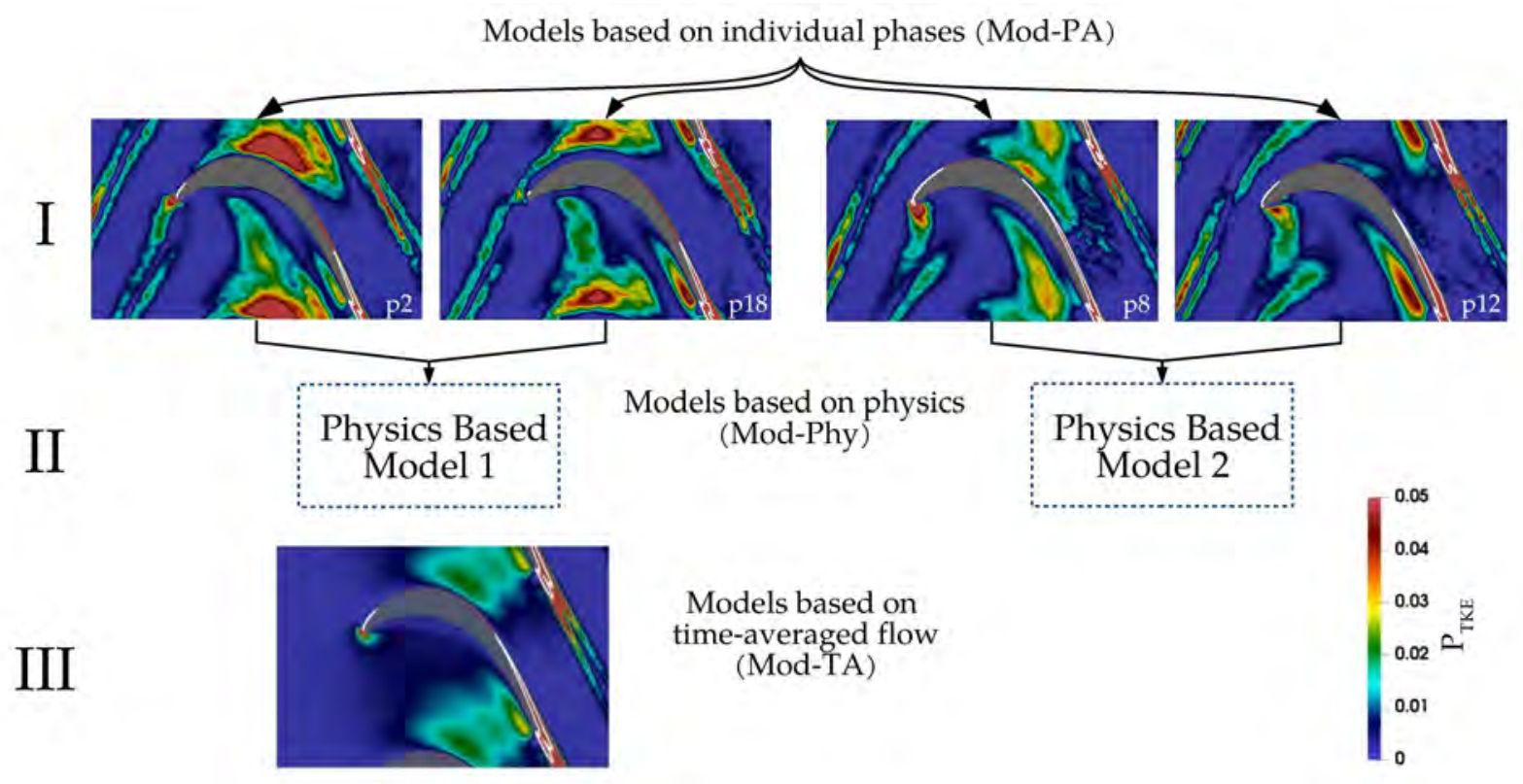

Fig. 4: The three approaches used for URANS EARSM development. Contour plots of TKE production for various phase-lock averaged and time-averaged flow fields are shown for the 1B2U case.

The wakes coming off the blade TE (TE wake region) are dominated by either higher diffusion due to the formation of KelvinHelmholtz roll-up vortices or non-linear phenomena due to intermediate wake and TE wake interactions. As noted by Lumley [36], it is challenging to develop a constitutive law that holds valid in regions with very different combinations of time and length scales. Therefore, it has been hypothesized that different EARSMs need to be produced for each of these three different regions.

Firstly, criteria need to be determined that allow objective separation of the different wake regions for which models will be trained. Analyzing the flow fields shows that there is a sharp increase in the strain rate magnitude due to the curvature effects of the LPT blade, approximately 5-7\% $\mathrm{C}$ upstream of the blade LE. This criterion can be used to mark the inception of the intermediate wake region and was inspired by a study conducted by Michelassi and Wissink [37]. They showed that the wake (intermediate wake in Fig. 3), when being swallowed into the blade passage, undergoes distortion and it responds to the alignment or misalignment of the strain rate tensor with the Reynolds stress tensor. The TE wake region can be easily identified due to higher TKE values and dissipation rates as compared to the surrounding wake passage. The near wake region (up to $5 \% \mathrm{C}$ in the streamwise direction from the blade TE) has been ignored from the TE wake training region as it drastically affects the nature of the trained models due to the high shear as compared to the rest of the (far) wake.

Model Types Using both the 1B2U and 2B3U cases, with their respective phase-lock averaged flow fields, three kinds of model types have been developed as shown in Fig. 4. In Fig. 4 , contours of TKE production are shown only for four phases of the $1 \mathrm{~B} 2 \mathrm{U}$ case and for the time-averaged flow field. There is an additional contour line shown in white for $\mathrm{P}_{T K E}=0.25$, which highlights the extent of laminar separation and its effect in the TE wake. The first model type uses the individual phase-lock averaged (Mod-PA) flow fields to generate models for each indi- vidual phase and training region. As there are three training regions and 20 and 17 phase-lock averaged data sets for the 1B2U and 2B3U cases, respectively, this implies that there will be 60 different models for the 1B2U case and 51 different models for the 2B3U case, post-ensemble averaging (i.e. the GEP algorithm was run a total 1500 times in order to obtain the 60 models for $1 \mathrm{~B} 2 \mathrm{U}$ case and it was run 1275 times to obtain 51 models for the 2B3U case). In order to assess whether a large number of models are required to be developed to predict the physics across all the phases and training regions, alternative methods which lead to a smaller number of models have been investigated. The second type of models are ones that are based on the wake physics (ModPhy). In Fig. 4, as an example, data from phases $2 \& 18$, along with a number of other phases (not shown in Fig. 4), which have similar physics, are combined together to form a larger dataset which is used for developing an EARSM. In this case, phases 8 $\& 12$ have different physics in the TE wake region as compared to the phases $2 \& 18$ and hence the data from phases $8 \& 12$, along with a number of other phases (not shown in Fig. 4), are used to develop a different EARSM. The criteria for the development of Mod-Phy models is discussed in later sections. The third model type uses the time-averaged statistics (Mod-TA) across one time period (i.e. no phase data) for EARSM development; similar to what was done for the cases with steady inflow conditions considered in Akolekar et al. [30]. The subsequent sections analyze the performance of the various models developed using the three different model development approaches.

\section{Models Based on Individual Phases}

This section discusses the models that have been generated using the Mod-PA approach. These models were developed for all three wake regions for both cases. However, it was found that the models developed in the TE wake region offer the most interesting trends and correlations to flow physics, and thus have been discussed in detail. An assessment of the performance of these 

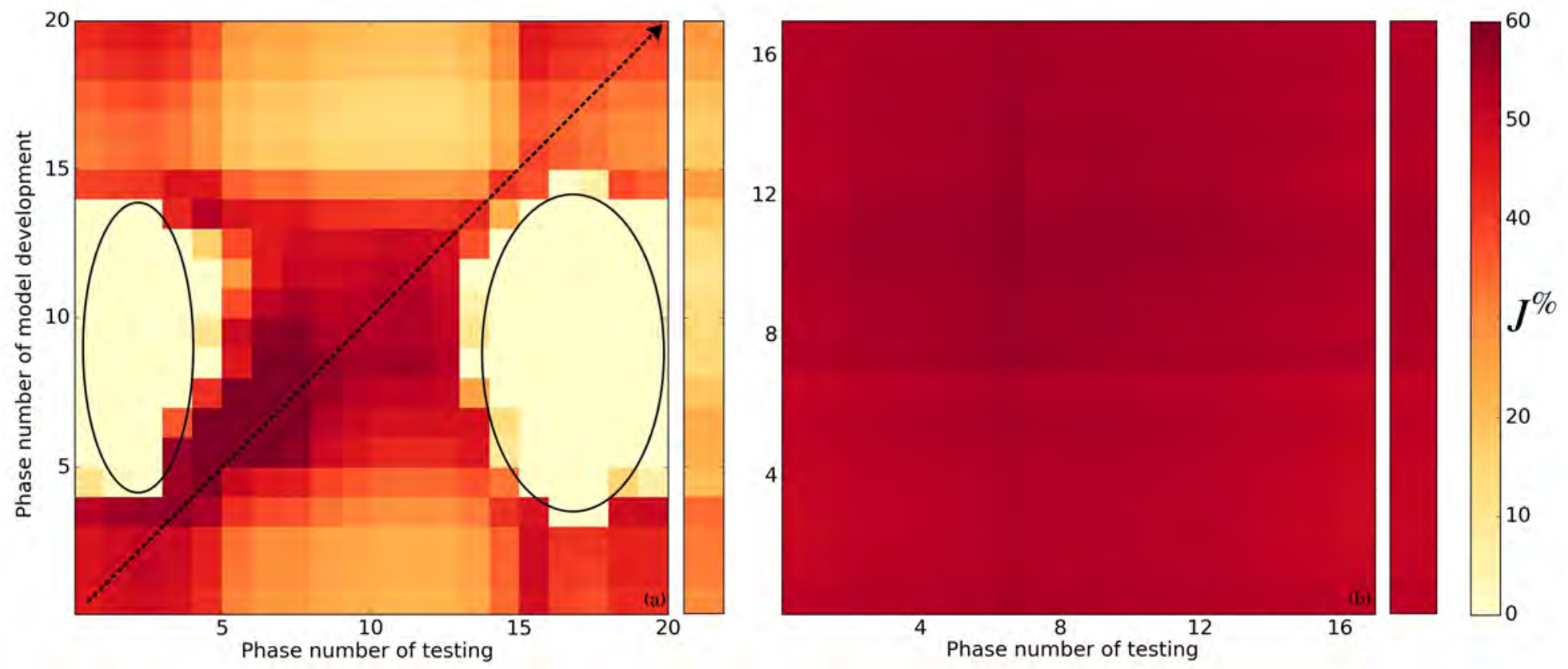

Fig. 5: Percentage reduction in normalized mean square error $\left(J^{\%}\right)$ over the Boussinesq approximation, when models developed on the TE wake region of a particular phase are tested on the TE wake region of all the phases for the (a) 1B2U and (b) 2B3U cases. The additional column on the right of each plot is the average $J^{\%}$ a model can bring if it is applied to all the phases.

models has been conducted in an a priori sense (without running any (U)RANS calculations). This will give designers some insights into how the developed models perform in the different flow regimes and what are the best practices for model generation and selection.

Error Reduction Matrices With the Mod-PA approach for model development, each model that is generated has been tested on each of the other phases for the respective training region. This will provide insight regarding the robustness of the models developed for each of the phases (i.e. do models trained on one particular phase perform well on the other phases). In order to achieve this, the normalized mean square error (Eq. 5) was calculated by testing each of the models developed ( 20 in the case of $1 \mathrm{~B} 2 \mathrm{U}$ and 17 in the case of $2 \mathrm{~B} 3 \mathrm{U}$ ) by the Mod-PA approach on each of the phase-lock averaged data sets for the respective training regions and cases. The normalized mean square errors using the Boussinesq approximation were also calculated (replace $\boldsymbol{a}_{i j}^{E A R S M}$ in Eq. 5 with $\boldsymbol{a}_{i j}^{B}$ ). The percentage difference between these two normalized mean square errors (or cost functions), is used to define an error metric as

$$
J^{\%}=\frac{\left(J^{B}-J^{E A R S M}\right)}{J^{B}} \times 100 .
$$

$J^{\%}$ shows how well a developed model performs over the Boussinesq approximation. It also shows how well the developed model represents the LES anisotropy field (for example, if $J^{\%}=100$, this implies that $J^{E A R S M}=0$ and the developed model fits the LES data perfectly). The percentage reduction in normalized mean square error $\left(J^{\%}\right)$, or simply the percentage error reduction, for the TE wake region has been plotted in Fig. 5 for both cases. The extra column on the right of these matrices depicts the average percentage error reduction a model can bring if it is applied to all the phases.

In Fig. 5(a) (1B2U case), there are two distinct light patches (demarcated by the ellipses) which represent either a zero or negative (which is also shown as zero for the ease of presentation) percentage reduction in normalized mean square error over the Boussinesq approximation. This implies that certain models, when used on other phases, do not improve the anisotropy prediction, but can in fact even worsen it. Broadly categorizing, models developed on phases 5-14 do not enhance performance when they are tested on phases 1-4 and 15-20. Models trained on phases 1-4 and 15-20, however, on the other hand, not only do offer significant percentage error reductions if they are tested on phases 1-4 and 15-20, but also offer some degree of error reduction for phases 5-14. Models trained on phases 5-14, when tested on phases 5-14 do offer a percentage reduction in normalized mean square error of an average value of approximately $53 \%$. Models developed on the respective phases of the TE wake region in the $2 \mathrm{~B} 3 \mathrm{U}$ case (Fig. 5 (b)), when tested on the other phases, offer a uniformly distributed percentage error reduction of $55 \%$ across all the phases. These observations can be explained if the physics of the boundary layer and the TE wake region are analyzed.

Boundary Layer Physics The incident wakes from previous blade rows interact with the suction side boundary layer in a number of ways. At moderate Reynolds numbers (inc. $\left.\operatorname{Re}_{2 i s}=100,000\right)$ and mild adverse pressure gradients, there is a possibility that laminar separation can occur on the blade suction side, which in fact happens in the 1B2U case. Based on the LES data [2] of the 1B2U case, it is observed that intermittent laminar separation (short laminar separation bubbles [38]), followed by turbulent reattachment, takes place. Due to the discrete incident wakes, in the 1B2U case, the shear layer on the aft-side of the suction side gets perturbed and separates. Due to 

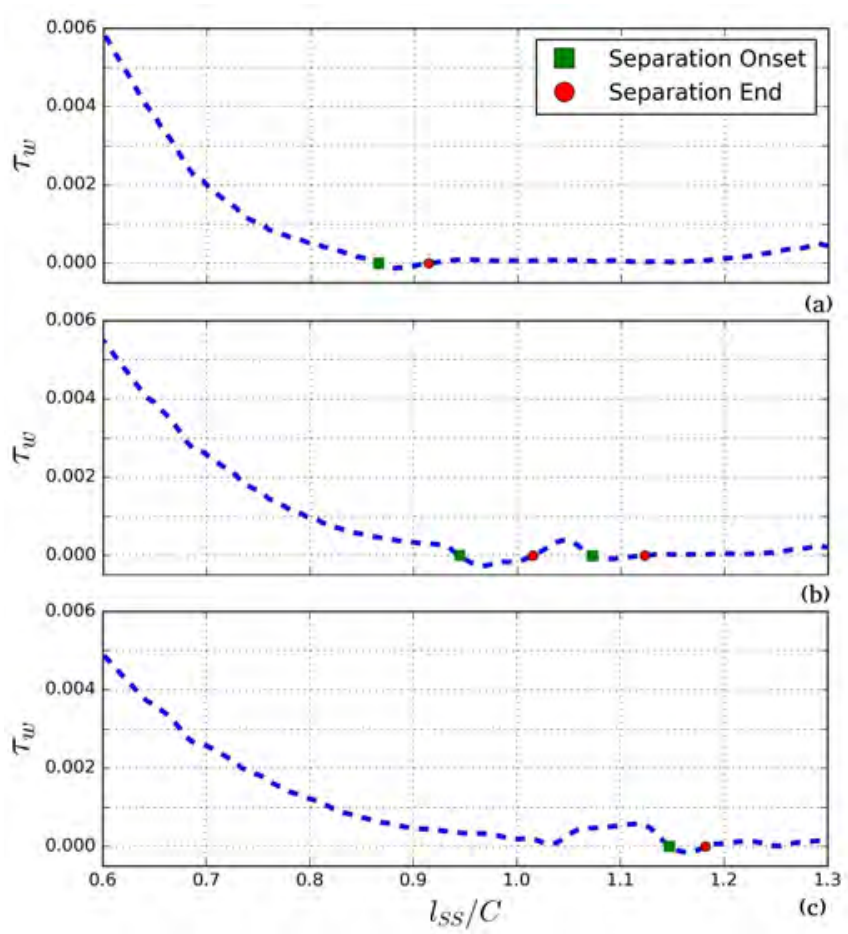

Fig. 6: Wall shear stress as a function of the normalized suction side surface length for phases (a) 5, (b) 8 and (c) 10, denoting the separation onset and end and highlighting the propagation of the separation bubble(s) for the 1B2U case.

these perturbations, Kelvin-Helmholtz roll-up vortices are [4,39] formed. These roll-up vortices perturb the boundary layer further downstream which leads to the formation of more vortices; eventually breaking down into turbulence, and thereby causing boundary layer transition. A calmed region soon follows once the roll-up vortices and turbulent boundary layer has subsided. Figure 4 shows the TKE production contours for phases ( $2 \& 18)$ which have calmed boundary layers and phases $(8 \& 12)$ where the Kelvin-Helmholtz roll-up vortices are shed.

Figure 6 depicts the wall shear stress as a function of the suction side surface length $\left(l_{S S}\right)$ normalized by the real chord for phases 5,8 , and 10, which highlights the propagation of short laminar separation bubbles along the suction side boundary layer of the 1B2U case. The area between the separation onset and end markers in Fig. 6, marks the regions where the wall shear stress is less than zero (separation). The separation bubble first appears in phase 5, and is also visible in phases 6-10. The separation becomes stronger in phase 8 , in which the flow separates and reattaches twice. By phase 10 the separation bubble has become weak, and it ceases to exist in subsequent phases. However, its effect can certainly be felt downstream of the TE in later phases. Due to the laminar separation, and boundary layer transition resulting in turbulent reattachment, there is an increase of turbulent diffusion in the boundary layer and thus in the TE wake region. Turbulence models using the Boussinesq approximation, generally cannot predict this change of diffusion across the various phases. However, with the EARSMs developed for the TE wake region, this increase in turbulent diffusion has been successfully captured.

The amount of additional diffusion required to predict the flow physics more accurately in the TE wake region for each of

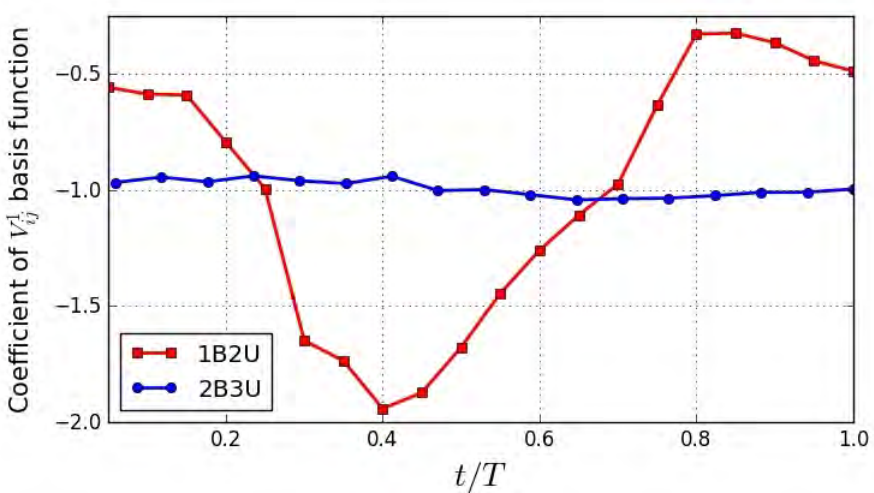

Fig. 7: Additional diffusion (coefficient of $\boldsymbol{V}_{i j}^{1}$ in $\boldsymbol{a}_{i j}^{E A R S M}$ ) required in the TE wake region of the $1 \mathrm{~B} 2 \mathrm{U}$ and $2 \mathrm{~B} 3 \mathrm{U}$ cases as predicted by the EARSM models for one time period.

the 20 phases is shown in Fig. 7 for both cases and for one time period. The additional diffusion is represented by the coefficient of the $V_{i j}^{1}$ tensor basis function. This diffusion is additional to the one that is already offered by the Boussinesq approximation from the $-\frac{v_{t}}{k} \boldsymbol{S}_{i j}^{\prime}$ term. It should be noted that the additional diffusion (coefficient) in Fig. 7 is represented by a negative number as the coefficient of $\boldsymbol{V}_{i j}^{1}$ is negative. As the diffusion in the TE wake region increases the coefficient of $\boldsymbol{V}_{i j}^{1}$ becomes increasingly negative. In Fig. 7 (and also in Fig. 8) models have been tested on the same phase that they were developed on (i.e. along the diagonal line in Fig. 5(a)). Note that data is available only at the markers, since only phase-lock averaged data is used. Lines between the markers are there to aid visual representation. From Fig. 7 it is clear that additional diffusion is required for all phases to obtain significant error reduction over the Boussinesq approximation. In the 1B2U case, phases 6-12 have an additional diffusion coefficient with a magnitude greater than 1.3 , as the boundary layer and TE wake region are perturbed by laminar separation. Since the effect of the laminar separation is convected at a speed of $50-90 \%$ of the so-called freestream fluid [39], there is a sharp increase in the additional diffusion coefficient in the TE wake region only in phase 6 , even though the laminar separation bubble is visible in phase 5. The magnitude of the coefficient of the $\boldsymbol{V}_{i j}^{1}$ tensor basis function gradually starts to fall from phase 8 and makes a sharp drop after phase 14, when the residual effects of separation and a perturbed suction side boundary layer have completely subsided. A relatively calm period follows the separation event and the suction side boundary layer remains laminar until phase 3 of the next time period; hence the lower additional diffusion coefficient. Some instabilities start to form again in phase 4. Since the additional diffusion coefficient changes drastically between the calm phases and phases where there is laminar separation, distinctive light patches are formed in Fig. 5(a), when models with higher additional diffusion coefficients are tested on models with lower additional diffusion coefficients. If models developed on phases which require lower additional diffusion (phases 1-4 \& 15-20) are tested on phases which require higher additional diffusion (5-14), they still reduce the error as they contribute to a fraction of the additional diffusion coefficient required to bring about the maximum possible error reduction.

In the 2B3U case, due to a larger number of bar wakes and higher bar convection velocity than the $1 \mathrm{~B} 2 \mathrm{U}$ case, there are no 
discrete wakes that are being formed. Consequently, the boundary layer does not have an opportunity to undergo laminar separation. Therefore, there is hardly any change in the additional diffusion coefficient across phases. Due to the higher convection speed of the incoming wakes, an additional diffusion coefficient of around -1 is still required to attain the maximum possible error reduction as outlined in Fig. 5(b). This also suggests that one EARSM is sufficient to model all the phases with a good degree of accuracy for the $2 \mathrm{~B} 3 \mathrm{U}$ case.

Intermediate Wake - Trailing Edge Wake Interactions Another interesting point to note is the percentage contribution of the non-linear terms (i.e. tensor basis functions $\boldsymbol{V}_{i j}^{2}, \boldsymbol{V}_{i j}^{3}$ and $\boldsymbol{V}_{i j}^{4}$ ) to the error reduction for the TE wake region. Figure 8 demonstrates the percentage contribution of non-linear terms to the net percentage error reduction when a model developed on a particular phase is tested on that same phase (i.e. along the diagonal line in Fig. 5(a)). In the 1B2U case, for phases 1-8, the contribu-

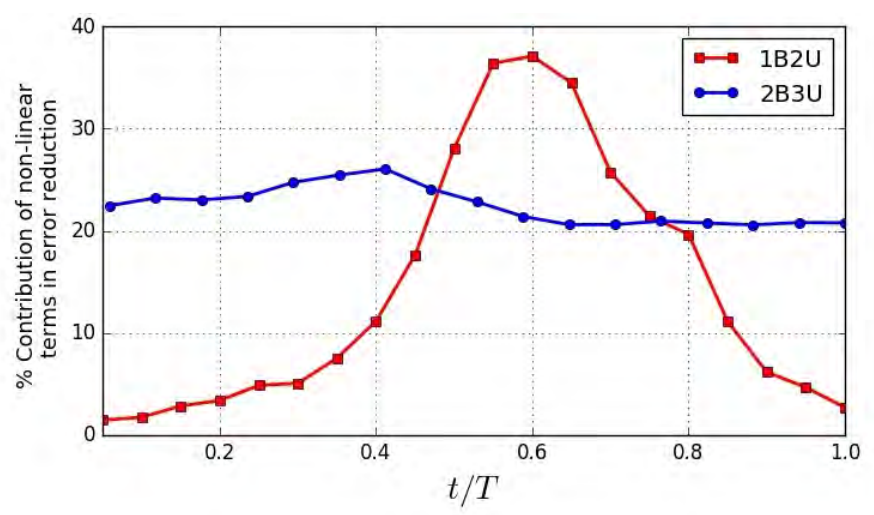

Fig. 8: Percentage contribution of non-linear terms to the net percentage normalized mean square error reduction $\left(J^{\%}\right)$ for the $1 \mathrm{~B} 2 \mathrm{U}$ and $2 \mathrm{~B} 3 \mathrm{U}$ cases in the TE wake region across one time period.

tion of the non-linear terms is less than $10 \%$, which implies that the TE profile wake is diffusion dominated. From phases 9 to 16 there is an increase in the contribution of the non-linear terms to the total error reduction. This contribution can be attributed to the wake, that was originally in the intermediate wake region, moving downstream and interacting with the wake in the TE region. Figure 9 shows TKE contour plots of phase-lock averaged flow fields for phases 3, 9, 12 and 20 for the 1B2U case. In phase 3 , the intermediate wake in the blade passage does not interact with the TE wake region; hence the very low contribution of the non-linear terms to the error reduction. The intermediate wake - TE wake interactions start to intensify in phase 9 and peak in phase 12 . By phase 18 , as the wake through the TE wake passage exits the domain, the effect of these interactions largely subsides, as is clearly visible from the TKE plot for phase 20 . Figure 10 shows the TKE contour plots for phases 7 and 15 for the $2 \mathrm{~B} 3 \mathrm{U}$ case. Note the different scaling used for the TKE contours (to highlight the wakes for this case). In the 2B3U case, since there are no discrete wakes that propagate through the blade passage, and since there is a near continuous wake-wake interaction, the contribution of the non-linear terms remains reasonably constant.

The phenomenon of wake-wake interactions is also observed in the incoming and intermediate wake regions of the 2B3U case. The percentage contribution of the non-linear tensor basis functions to the net percentage reduction in the normalized mean square error $\left(J^{\%}\right)$ is much higher in the incoming wake region than the TE wake region of the $2 \mathrm{~B} 3 \mathrm{U}$ case. This can can be attributed to stronger wake-wake interactions in the incoming wake region of the $2 \mathrm{~B} 3 \mathrm{U}$ case.

\section{Models Based on Physics \& Time Averages}

It is not convenient for anyone to run different models for each of the phases. Therefore, the performance of models of Mod-Phy and Mod-TA types (Fig. 4) have been assessed with respect to the Mod-PA approach, as these model types consist of a smaller number of models. In a machine-learning context, it is assumed that a model developed on a particular dataset should perform the best when it is applied to that same dataset (Mod-PA approach). The datasets from the Mod-PA approach serve as test data for the models generated from the Mod-Phy and Mod-TA approaches. It is thus desirable to develop Mod-Phy \& Mod-TA models that offer comparable performance to the Mod-PA models. For the TE wake region in the $1 \mathrm{~B} 2 \mathrm{U}$ case, based on the insight gained from Figs. 5(a), 7 and 8 in regard to amount of additional diffusion and intensity of intermediate-TE wake interactions across each of the phases, two physics based models have been created: one comprising data from phases 5-14 and the other comprising data from phases $1-4 \& 15-20$. For the $2 \mathrm{~B} 3 \mathrm{U}$ case, only one physics based models is created due to the similar nature of flow physics across all the phases. Equations of the developed models using the Mod-Phy approach for the TE wake region for the $1 \mathrm{~B} 2 \mathrm{U}$ and $2 \mathrm{~B} 3 \mathrm{U}$ cases are presented below. Only the linear portion of the coefficients of the tensor basis function are provided (i.e. products of invariants are neglected as they are negligible):

1B2U-Mod-Phy: Phases 1-4 \& 15-20:

$$
\begin{aligned}
\boldsymbol{a}_{i j}^{\text {EARSM }} & =-\frac{v_{t}}{k} \boldsymbol{S}_{i j}^{\prime}+\left(-0.448+0.094 I_{1}+0.005 I_{2}\right) \boldsymbol{V}_{i j}^{1}+(0.387 \\
& \left.-0.14 I_{1}+0.947 I_{2}+0.387\right) \boldsymbol{V}_{i j}^{2}+\left(1.036-0.758 I_{1}\right. \\
& \left.+0.853 I_{2}\right) \boldsymbol{V}_{i j}^{3}+\left(-0.87+1.6 I_{1}-0.6 I_{2}\right) \boldsymbol{V}_{i j}^{4} .
\end{aligned}
$$

1B2U-Mod-Phy: Phases 5-14:

$$
\begin{aligned}
\boldsymbol{a}_{i j}^{E A R S M} & =-\frac{v_{t}}{k} \boldsymbol{S}_{i j}^{\prime}+\left(-1.39+12.648 I_{1}+6.641 I_{2}\right) \boldsymbol{V}_{i j}^{1}+(4.66 \\
& \left.-13.304 I_{1}\right) \boldsymbol{V}_{i j}^{2}+\left(5.097-9.776 I_{1}+10.476 I_{2}\right) \boldsymbol{V}_{i j}^{3} \\
& +\left(-8+15.606 I_{1}-48.9 I_{2}\right) \boldsymbol{V}_{i j}^{4} .
\end{aligned}
$$

2B3U-Mod-Phy:

$$
\begin{aligned}
\boldsymbol{a}_{i j}^{E A R S M} & =-\frac{v_{t}}{k} \boldsymbol{S}_{i j}^{\prime}+\left(-1.11+9.784 I_{1}+2.613 I_{2}\right) \boldsymbol{V}_{i j}^{1}+(-1.198 \\
& \left.+2.215 I_{1}+16.066 I_{2}\right) \boldsymbol{V}_{i j}^{2}+\left(5.8-22.575 I_{1}+34.15 I_{2}\right) \boldsymbol{V}_{i j}^{3} \\
& +\left(-7.608+40.486 I_{1}-44.913 I_{2}\right) \boldsymbol{V}_{i j}^{4} .
\end{aligned}
$$

The following section offers a comparison between the three model types. 

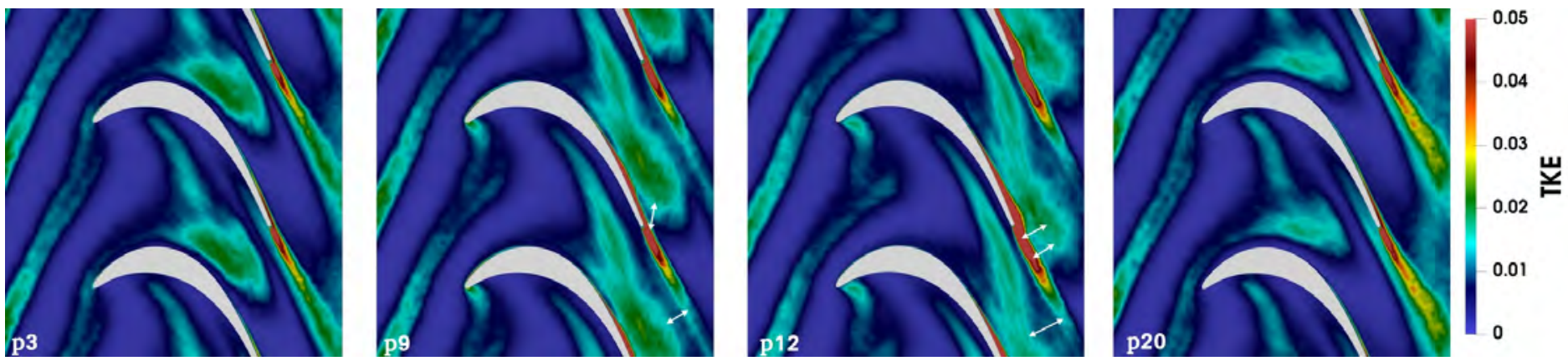

Fig. 9: Phase-lock averaged TKE contour plots highlighting the movement of the intermediate wakes through the blade passage and their interaction, shown by the white arrows, with the TE profile wakes, for the 1B2U case at phases (a) 3, (b) 9, (c) 12 and (d) 20.
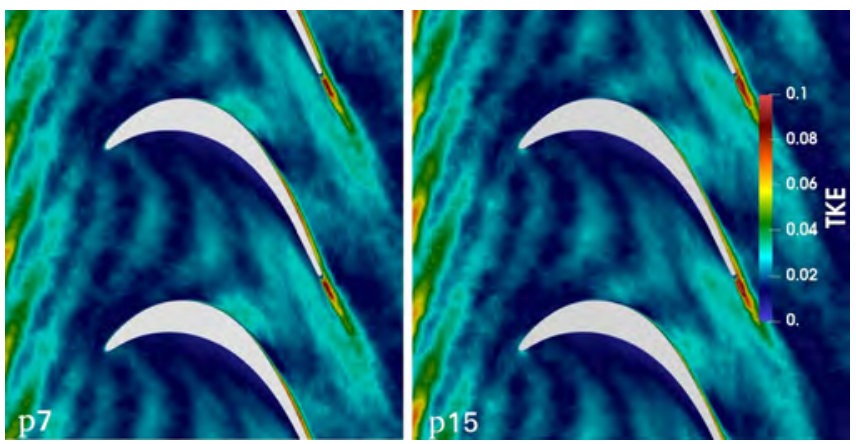

Fig. 10: Phase-lock averaged TKE contour plots for the 2B3U case at phases (a) 7 and (b) 15 .

\section{Comparison of Models}

This section compares the three model development approaches in terms of model coefficient, $J$, shear stress profiles and offers general guidelines of the range of applicability of the models developed across all three wake regions in Fig. 3.

Model Coefficients \& Error Reduction Figure 11 represents the coefficients of the $\boldsymbol{V}_{i j}^{1}$ and $\boldsymbol{V}_{i j}^{4}$ tensor basis functions in the TE wake region for the (a) 1B2U and (b) 2B3U cases, when models developed using the Mod-PA, Mod-Phy and ModTA approaches are tested on each of the phase-lock averaged datasets for both cases. The percentage reduction in the normalized mean square error $\left(J^{\%}\right)$ is also shown. In the 1B2U case, the 1B2U-Mod-TA model does not seem to determine the tensor basis functions' coefficients accurately when compared to the models developed using the 1B2U-Mod-PA approach as the effect of laminar separation bubbles is washed out due to the time averaging process. Due to time averaging, some essential flow features can be removed and this neutralizes the effect of certain flow physics [40]. Therefore, what remains in the time-average is just an attached boundary layer which offers an average additional diffusion coefficient of -0.57 and a $\boldsymbol{V}_{i j}^{4}$ coefficient of 3.9 , which is quite different from the coefficients in phases 5-14 where laminar separation is seen. For certain phases, this model gives a negative reduction (shown as zero in Fig. 11(a)) in the mean square error which can be attributed to the improper determination of all the coefficients of the tensor basis functions. For the 2B3U case, the model 2B3U-Mod-TA offers much better performance than its corresponding $1 \mathrm{~B} 2 \mathrm{U}$ case, as there is a much smaller variance in the physics that is occurring between different phases. There is still, however, a relatively small neutralizing effect of certain flow physics in the time-averaged flow field which results in slightly higher coefficients of some of the tensor basis functions. This accounts for the marginal difference in the normalized mean square error as compared to the models developed on individual phases (2B3U-Mod-PA).

In both the cases, the models that were developed using the Mod-Phy approach determine the coefficients of the tensor basis functions closely resembling those from the Mod-PA approach. They also offer similar percentage error reduction as compared to the Mod-PA approach. In the 1B2U case, the hypothesis of using 2 Mod-Phy (1B2U) models seems the correct way of approaching the problem, as it has captured the coefficients of the tensor basis functions in phases 1-4 \& 15-20 quite closely when compared with the coefficients obtained using the 1B2U-ModPA models. The value of the tensor basis functions' coefficients for phases 5-14, derived via the Mod-Phy approach, appears like a weighted average value of the coefficients of the tensor basis functions in phases 5-14 from the Mod-PA approach. At the junction where one model is switched on and the other switched off (phases $4 \& 15$ in the 1B2U case) there is a slight drop in the percentage mean square error reduction. This issue can be alleviated by using a ramping function in order to switch between the two Mod-Phy models during URANS implementation. This will either eliminate or alleviate the slope of the kink in the percentage error reduction plot around phases $4 \& 15$. The 2B3U-ModPhy model also performs equally well or slightly better when compared with the 2B3U-Mod-PA models, for the 2B3U case.

From the perspective of model versatility, it has been observed that the 2B3U-Mod-Phy model, when applied to the TE wake region of phases 5-14 offers an average percentage error reduction $\left(\mathrm{J}^{\%}\right)$ of $37 \%$, whereas the 1B2U-Mod-Phy model developed on phases 5-14, and tested on the TE wake region of the 2B3U case offers an average percentage error reduction $\left(J^{\%}\right)$ of $45 \%$. This shows that the models can be interchangeably used in regions of similar physics for different operating conditions.

Mod-TA Models The Mod-TA model type, in the context of cases with unsteady inflow conditions, has limited applicability for model development, as some of the underlying phenomena related to deterministic unsteadiness is neutralized due to time averaging [40]. As shown in Fig. 11(a), if there is variance in the boundary layer physics, these models offer negative or zero improvement over the Boussinesq approximation, for some phases in the TE wake region. Due to the time-averaging process, there are no distinct incoming or intermediate wakes in the time-averaged flow field (refer to Fig. 4), i.e. the wakes are averaged out. Therefore, the Mod-TA approach cannot yield ef- 

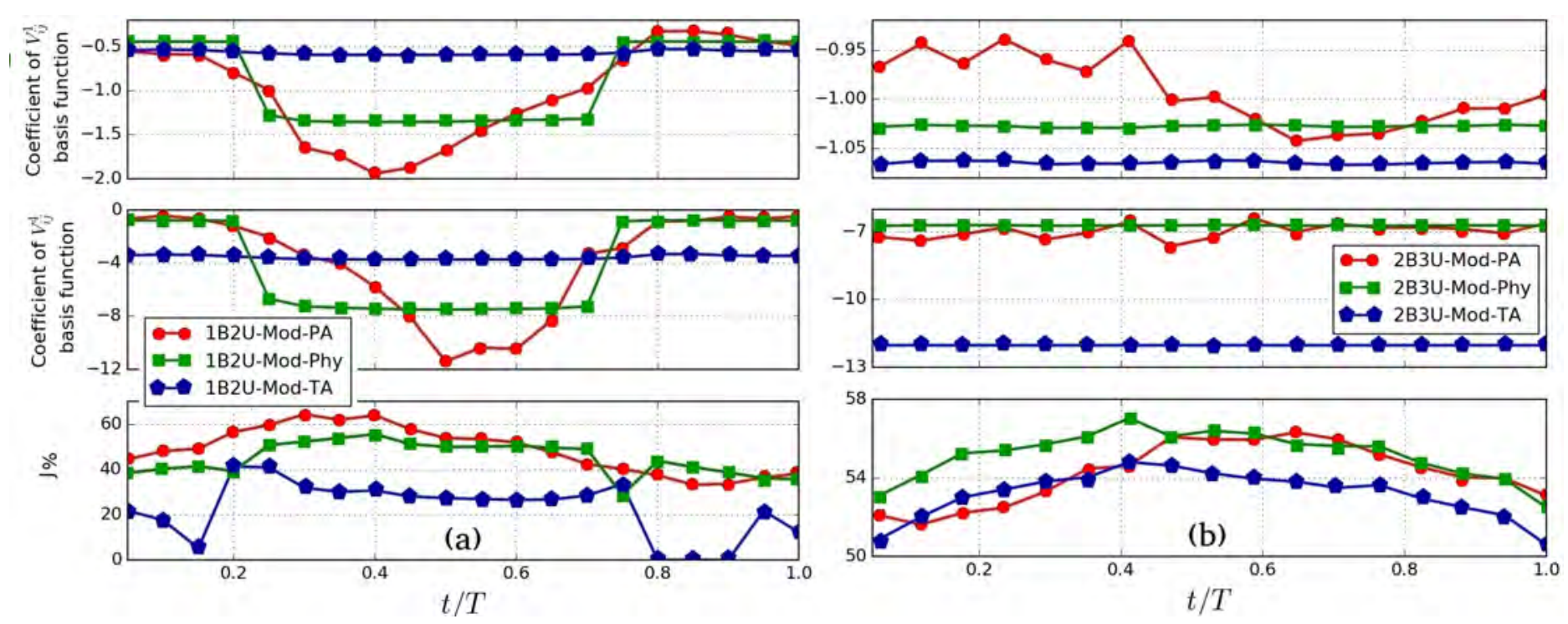

Fig. 11: Coefficients of $\boldsymbol{V}_{i j}^{1}$ and $\boldsymbol{V}_{i j}^{4}$ tensor basis functions and percentage reduction in normalized mean square error $\left(J^{\%}\right)$ in the TE wake region of the (a) 1B2U \& (b) 2B3U cases, when models developed using the Mod-PA, Mod-Phy and Mod-TA approaches are tested on phase-lock averaged data across one time period.

fective models for the incoming and intermediate wake regions. They can however be used for the model development in the TE region for a case which has a high reduced frequency (i.e. presence of wake fogging) with a good degree of reliability. Due to their limited applicability, these models have been excluded from the comparison study of Reynolds shear stress profiles in Fig. 12.

Mod-Phy Models Mod-Phy models offer comparable, or in some cases slightly better performance not only for the TE wake regions, as shown in Fig. 11, but also for the incoming and intermediate wake regions, when compared with the Mod-PA approach for the respective region and case. The incoming and intermediate wake regions of the $1 \mathrm{~B} 2 \mathrm{U}$ case require only one Mod-Phy model per wake region as opposed to the two ModPhy models that are required in the TE wake region of the 1B2U case. This is because there is no significant variance in physical phenomena across phases. Therefore, the error reduction matrix of both the incoming and intermediate wake regions for the $1 \mathrm{~B} 2 \mathrm{U}$ case is qualitatively similar to Fig. 5(b). The formulation of the models developed with the Mod-Phy approach for the intermediate and incoming wake regions of the 2B3U case are quite similar. Therefore, one Mod-Phy model can be used to enhance the anisotropy prediction across both of these regions. This, however, does not hold for the 1B2U case, as, due to the discrete nature of the wakes, different physics dominate in the incoming wake and intermediate wake regions. This implies that four and two Mod-Phy based models are required to enhance the wake mixing prediction for the entire LPT domain of the 1B2U and 2B3U cases respectively. The developed Mod-Phy models bring about an average percentage reduction in normalized mean square error over the Boussinesq approximation by $45 \%$ across all three wake regions and both the cases.

It was also observed that Mod-Phy models developed on any of the wake regions of the $1 \mathrm{~B} 2 \mathrm{U}$ case (discrete wakes) when tested on the same regions of the 2B3U case (wake fogging) perform better than the Mod-Phy models that were developed on any of the wake regions of the 2B3U case and tested on the same regions of the 1B2U case. This shows that models developed on cases with discrete wakes are more robust. It is anticipated that since the Mod-Phy models developed on the 1B2U case perform well on the $2 \mathrm{~B} 3 \mathrm{U}$ case, which has a largely different reduced frequency and flow coefficient, these should also perform well for cases with different (smaller range than 2B3U) reduced frequencies and flow coefficients, such as $1 \mathrm{~B} 1 \mathrm{U}, 1 \mathrm{~B} 3 \mathrm{U}$ and $2 \mathrm{~B} 1 \mathrm{U}$ cases [2], which are also of interest to blade designers.

Shear Stress Prediction If the wake mixing prediction is to be enhanced, it is important that the prediction of the $\boldsymbol{\tau}_{x y}$ component of the Reynolds stress tensor is enhanced as compared to the Boussinesq approximation. Leschziner [41] states that in the numerical solution to many practical shear-driven flows, the crucial factor affecting predictive realism is the ability of the model to return the correct level of shear stress components. This implies if the newly developed models offer a better prediction of $\boldsymbol{\tau}_{x y}$ than the Boussinesq approximation, when compared with the actual value of $\tau_{x y}$ from a high-fidelity database, one can confidently say that the wake mixing prediction is enhanced in an $a$ priori sense.

Using the eddy viscosity calculated via a least square optimized approach [29], shear stress profiles, calculated based on LES data, from the three wake regions and different phases have been shown in Fig. 12 for the 1B2U case. The shear stress profiles obtained via the LES data (reference), Boussinesq approximation, Mod-PA and Mod-Phy models (for the respective phase and region) are shown. Shear stress profiles from the Mod-TA approach are not included as it is not a practical approach for the 1B2U case. The light grey region in these figures depicts where the EARSMs have been switched on (which is similar to the respective training region in Fig. 3; note that the movement of the wakes cause the training regions to slightly change across phases). At other locations, the Boussinesq approximation is used. The locations where these profiles are extracted are shown by the dashed lines in Fig. 3. Figure 12(a) shows $\tau_{x y}$ profiles $20 \% \mathrm{C}$ upstream of the blade LE for phase 3. In this case, the Mod-Phy and Mod-PA models offer comparable performance with respect to one another. These models bring about significant 

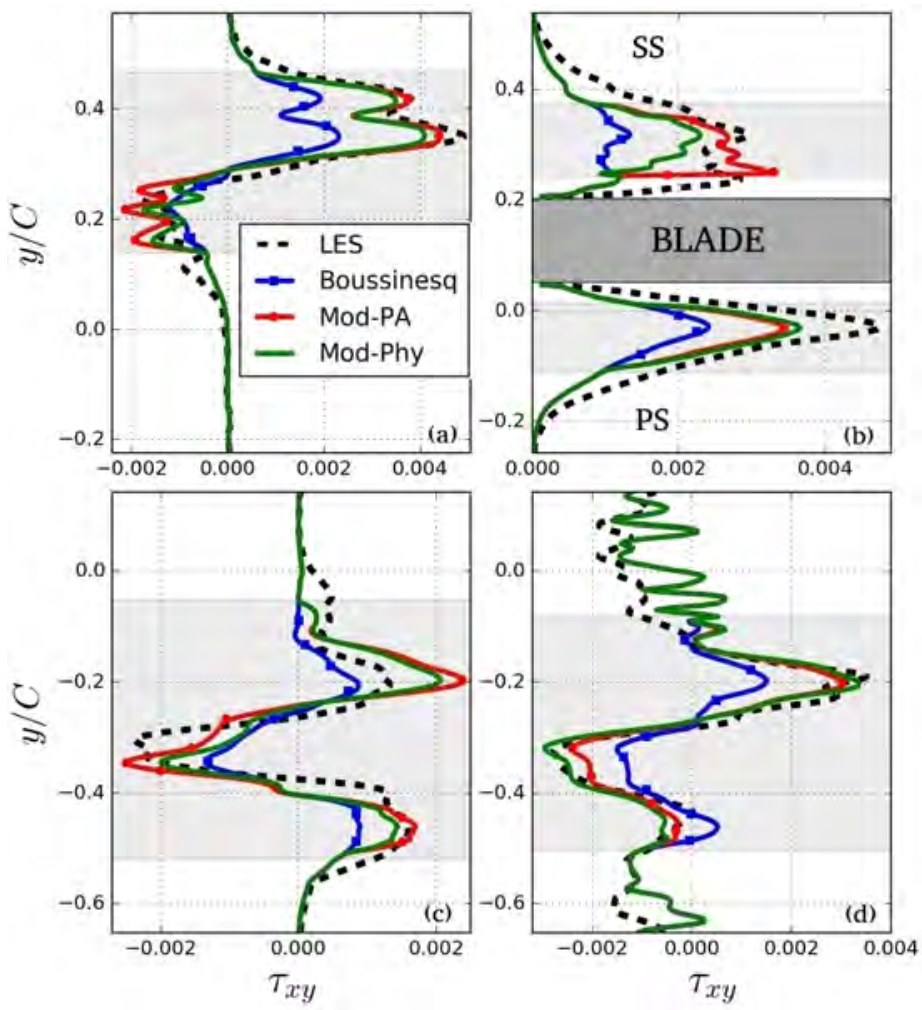

Fig. 12: Reynolds shear stress profiles for (a) p3, incoming wake region, (b) p6 intermediate wake region, (c) p9, TE wake region and (d) p16, TE Wake region for the 1B2U case. Data extraction locations are shown in Fig. 3.

improvement in $\tau_{x y}$ as compared to the Boussinesq approximation as they reduce the $\boldsymbol{\tau}_{x y}$ peak prediction error at $y / C=0.35$ and $y / C=0.43$ with respect to the LES from $50 \%$ (error due to Boussinesq approx.) to $8.5 \%$ (Mod-PA approach). Similar observations have been made for the intermediate wake region (Fig. 12(b)), which shows shear stress profiles along a vertical line $35 \% \mathrm{C}$ downstream of the blade LE. In this case, the ModPA model in phase 6 does slightly better on the suction side of the intermediate wake than the Mod-Phy model. On the pressure side the performance of these two models is comparable.

In the TE wake region (profiles are extracted 30\%C downstream of the blade TE), both the Mod-Phy models obtained are tested on the corresponding phases. Figure 12(c) shows the $\tau_{x y}$ profile using the Mod-Phy model with higher additional diffusion (Eq. 8), tested on phase 9. Overall, the Mod-Phy model performs quite well as compared to the Mod-PA model and brings about significant improvements in $\tau_{x y}$ over the Boussinesq approximation. In phase 16 of the TE wake (Fig. 12(d)), the Mod-Phy model with lower additional diffusoion (Eq. 7) traces out the LES stress profile quite accurately, and in fact performs better than the Mod-PA model developed on the TE wake of phase 16. In the 2B3U case (not shown here), the Mod-Phy models offer comparable performance with the Mod-PA models across all wake regions and outperform the Boussinesq approximation in terms of a priori shear stress predictions. These profiles consolidate the finding that the Mod-Phy models have the potential to offer a comparable, or at times, even slightly better performance as compared to a corresponding model that was developed using the individual phases (Mod-PA). Similar observations have also been made for other secondary statistics such as the other
Reynolds stress components and TKE production, but are not shown here for the sake of brevity. Since the newly developed models have enhanced the prediction of the shear stress and other secondary statistics by reducing the amount of normalized mean square error over the Boussinesq approximation, the wake mixing prediction has been enhanced.

\section{Conclusions}

This study explored methods to improve the URANS prediction of wake mixing in LPTs with unsteady inflow conditions, by creating a framework for the development of EARSMs using a machine learning approach, GEP. The EARSMs were developed using an LES database at an isentropic exit Reynolds number of 100,000 at two operating conditions. A zonal based model development approach was proposed. Therefore, EARSMs were developed for three wake regions deploying three distinct model development approaches, namely: using individual phase-lock averaged data (Mod-PA), using data from a combination of phases with similar physics (Mod-Phy), and using the timeaveraged statistics (Mod-TA). Phase-lock averaged data has provided valuable insights for model development which the timeaveraged data has failed to do across a broad spectrum of cases. Time-averaged data has limited applicability to extract insights from for cases with unsteady inflow as the time averaging process removes underlying physical phenonmena related to deterministic unsteadiness.

Using data from individual phases (Mod-PA approach), a detailed analysis of the wake physics to aid EARSM development was conducted for all the wake regions, with the main focus on the TE wake region. Based on a newly developed error metric, error reduction matrices were formed in order to assess the robustness of models across different phases. The trends in these matrices were explained by correlations drawn between the models developed and the flow physics. In the TE wake region of the cases with discrete incoming wakes there is a significant change of physics across phases. In order to capture these physics two Mod-Phy based EARSMs were proposed. For wake regions where the flow physics do not vary across the phases, one Mod-Phy model was found to be sufficient to enhance the wake mixing prediction. Therefore, one Mod-Phy model each was proposed for the incoming and intermediate wake regions, taking the total up to four Mod-Phy models for cases with discrete incoming wakes. For cases with non-discrete incoming wakes, since there is no significant variance of physics between the phases in the TE wake region, one Mod-Phy model is suffcient to enhance the wake mixing prediction in that region. These cases only require one model between the incoming and intermediate wake regions which takes the total to two Mod-Phy models for the entire flow domain. Based on the developed error metric, Mod-Phy models have significantly-reduced the error over the Boussinesq approximation by an average value of $45 \%$ across both cases and three wake regions, thus enhancing the wake mixing prediction.

This paper thus highlights the need for developing different non-linear turbulence closures to capture distinct physical phenomena accurately. The immediate challenge is to implement these models into URANS calculations with dynamic spatial and temporal blending functions to assess their performance over a wide range of operating conditions. 


\section{Acknowledgements}

This work was supported by the resources provided by the Pawsey Supercomputing Centre with funding from the Australian Government and the Government of Western Australia The support by the Australian Government Research Training Program Scholarship is acknowledged. The University of Melbourne authors also acknowledge the financial support and the permission to publish by General Electric.

\section{Nomenclature}

$\boldsymbol{a}_{i j} \quad$ Normalized anisotropy tensor

$C \quad$ Blade chord length

$F_{\text {red }} \quad$ Reduced frequency

$I_{k} \quad$ Scalar invariant

$J \quad$ GEP cost function

$J^{\%} \quad$ Percentage error reduction

$k \quad$ Turbulent kinetic energy

$l_{S S} \quad$ Blade suction side surface length

$M \quad$ Total number of training points

$N \quad$ Total number of phases

$p \quad$ Phase number

$P_{\text {bar }} \quad$ Bar pitch

$P_{T K E} \quad$ TKE production

$R e_{2 i s} \quad$ Isentropic exit Reynolds number

$S_{i j} \quad$ Strain rate: $\frac{1}{2}\left(\partial_{x_{j}} U_{i}+\partial_{x_{i}} U_{j}\right)$

$\boldsymbol{S}_{i j}^{\prime} \quad$ Deviatoric component of strain rate: $\boldsymbol{S}_{i j}-\frac{1}{3} \boldsymbol{\delta}_{i j} \boldsymbol{S}_{k k}$

$t \quad$ Time

$T \quad$ Time period

$U \quad$ Bar tangential speed

$\overline{v_{2}} \quad$ Wall-normal turbulent velocity fluctuations

$V_{2} \quad$ Exit absolute flow velocity

$\boldsymbol{V}_{i j}^{m} \quad$ Tensor basis function

$V_{x} \quad$ Axial velocity

$\boldsymbol{\delta}_{i j} \quad$ Kronecker Delta: $\boldsymbol{\delta}_{i j}=0$ if $i \neq j, \boldsymbol{\delta}_{i j}=1$ if $i=j$

$\zeta_{m} \quad$ Coefficient of $\boldsymbol{V}_{i j}^{m}$

$\mu_{t} \quad$ Eddy viscosity

$\rho \quad$ Density

$\tau \quad$ Turbulent time scale: $(1 / \omega)$

$\tau_{i j} \quad$ Reynolds stress tensor: $\rho \overline{u_{i}^{\prime} u_{j}^{\prime}}$

$\tau_{w} \quad$ Wall shear stress

$\Phi \quad$ Flow coefficient

$\omega \quad$ Specific dissipation rate

$\boldsymbol{\Omega}_{i j} \quad$ Rotation rate tensor: $\frac{1}{2}\left(\partial_{x_{j}} U_{i}-\partial_{x_{i}} U_{j}\right)$

\section{Acronyms}

$\begin{array}{ll}\text { B } & \text { Boussinesq } \\ \text { DNS } & \text { Direct numerical simulation } \\ \text { EARSM } & \text { Explicit algebraic Reynolds stress model } \\ \text { GEP } & \text { Gene expression programming } \\ \text { LE } & \text { Leading edge } \\ \text { LES } & \text { Large eddy simulation } \\ \text { LPT } & \text { Low-pressure turbine } \\ \text { Mod-PA } & \text { Models based on individual phases } \\ \text { Mod-Phy } & \text { Models based on physics } \\ \text { Mod-TA } & \text { Models based on the time-averaged flow }\end{array}$

RANS Reynolds averaged Navier-Stokes

SS Suction side

TE Trailing edge

TKE Turbulent kinetic energy

URANS Unsteady RANS

\section{REFERENCES}

[1] Schobeiri, M. T., and Ozturk, B., 2004. "Experimental Study of the Effect of Periodic Unsteady Wake Flow on Boundary Layer Development, Separation, and Re-attachment Along the Surface of a Low Pressure Turbine Blade”. In ASME Turbo Expo, pp. GT2004-53929.

[2] Michelassi, V., Chen, L., Pichler, R., Sandberg, R., and Bhaskaran, R., 2016. "High-Fidelity Simulations of Low-Pressure Turbines: Effect of Flow Coefficient and Reduced Frequency on Losses". J. Turbomach., 138(11)(November), p. 111006.

[3] Arndt, N., 1993. "Blade Row Interaction in a Multistage Low-Pressure Turbine". J. Turbomach., 115(1), p. 137.

[4] Stieger, R. D., and Hodson, H. P., 2004. "The Transition Mechanism of Highly-Loaded LP Turbine Blades". J. Turbomach., pp. 536-543.

[5] Stieger, R. D., and Hodson, H. P., 2005. "The Unsteady Development of a Turbulent Wake Through a Downstream Low-Pressure Turbine Blade Passage". J. Turbomach., 127(2), p. 388.

[6] Halstead, D. E., Wisler, D. C., Okiishi, T. H., Walker, G. J., Hodson, H. P., and Shin, H. W., 1997. "Boundary Layer Development in Axial Compressors and Turbines : Part 3 of 4 - LP Turbines”. J. Turbomach., 119(April), pp. 225-237.

[7] Michelassi, V., Chen, L. W., Pichler, R., and Sandberg, R. D., 2015. "Compressible Direct Numerical Simulation of Low Pressure Turbine - Part II: Effect of Inflow Disturbances". J. Turbomach., 137(7), p. 71005.

[8] Ranjan, R., Deshpande, S. M., and Narasimha, R., 2017. "New Insights from High-Resolution Compressible DNS Studies on an LPT Blade Boundary Layer". Comput. Fluids, 153, pp. 49-60.

[9] Wissink, J. G., 2003. "DNS of Separating, Low Reynolds Number Flow in a Turbine Cascade with Incoming Wakes". Int. J. Heat Fluid Flow, 24(4), pp. 626-635.

[10] Michelassi, V., Wissink, J. G., and Rodi, W., 2003. "Direct Numerical Simulation, Large Eddy Simulation and Unsteady Reynolds-Averaged NavierStokes Simulations of Periodic Unsteady Flow in a Low-Pressure Turbine Cascade: A Comparison”. Proc. Inst. Mech. Eng. Part A J. Power Energy, 217(4), pp. 403-411.

[11] Praisner, T. J., Clark, J. P., Nash, T. C., Rice, M. J., and Grover, E. A., 2006. "Performance Impacts Due to Wake Mixing in Axial Flow Turbomachinery”. In ASME Turbo Expo, no. GT2006-90666.

[12] Keadle, K., and Mcquilling, M., 2013. "Evaluation of RANS Transition Modeling for High Lift LPT Flows at Low Reynolds Number". In Proc. ASME Turbo Expo 2013 Turbine Tech. Conf. Expo., pp. GT2013-95069.

[13] Pacciani, R., Marconcini, M., Arnone, A., and Bertini, F., 2013. "Predicting High-Lift Low-Pressure Turbine Cascades Flow Using Transition-Sensitive Turbulence Closures". J. Turbomach., 136(5), p. 51007.

[14] Marconcini, M., Pacciani, R., Arnone, A., Michelassi, V., Pichler, R., Zhao, Y., and Sandberg, R., 2019. "LES and RANS Analysis of the End-Wall Flow in a Linear LPT Cascade with Variable Inlet Conditions, Part II: Loss Generation". J. Turbomach., 141(5), pp. 1-10.

[15] Schmitt, G., 2007. “About Boussinesq's Turbulent Viscosity Hypothesis: Historical Remarks and a Direct Evaluation of its Validity.". Comptes Rendus Mec. Elsevier Masson, 335(9), pp. 617-627.

[16] Pope, S. B., 1975. “A More General Effective-Viscosity Hypothesis”. J. Fluid Mech., 72(2), pp. 331-340.

[17] Gatski, T., and Speziale, C., 1993. "On Explicit Algebraic Stress Models for Complex Turbulent Flows". J. Fluid Mech., 254, pp. 59-78.

[18] Wallin, S., and Johansson, A. V., 2000. "An Explicit Algebraic Reynolds Stress Model for Incompressible and Compressible Turbulent Flows". J. Fluid Mech., 403, pp. 89-132.

[19] Duraisamy, K., Iaccarino, G., and Xiao, H., 2018. “Turbulence Modeling in the Age of Data". Annu. Rev. Fluid Mech.(March).

[20] Duraisamy, K., Zhang, Z. J., and Singh, A. P., 2015. "New Approaches in Turbulence and Transition Modeling Using Data-driven Techniques". In 53rd AIAA Aerosp. Sci. Meet., Vol. 1284. 
[21] Singh, A. P., Medida, S., and Duraisamy, K., 2017. "Machine-LearningAugmented Predictive Modeling of Turbulent Separated Flows over Airfoils". AIAA J., 55(7), pp. 2215-2227.

[22] Ling, J., Templeton, J., and Kurzawski, A., 2016. "Reynolds Averaged Turbulence Modelling Using Deep Neural Networks with Embedded Invariance". J. Fluid Mech, 807(2016), pp. 155-166.

[23] Wang, J.-X., Wu, J.-L., and Xiao, H., 2017. "A Physics Informed Machine Learning Approach for Reconstructing Reynolds Stress Modeling Discrepancies Based on DNS Data". Phys. Rev. Fluids, 2(3), p. 034603.

[24] Wu, J.-L., Xiao, H., and Paterson, E., 2018. "Physics-Informed Machine Learning Approach for Augmenting Turbulence Models: A Comprehensive Framework". Phys. Rev. Fluids, 3(July), p. 074602.

[25] Milani, P. M., Ling, J., and Eaton, J. K., 2019. "Physical Interpretation of Machine Learning Models Applied to Film Cooling Flows". J. Turbomach., 141(1), p. 011004.

[26] Ferreira, C., 2001. "Gene Expression Programming: a New Adaptive Algorithm for Solving Problems”. Complex Syst., 13(2), pp. 87-129.

[27] Weatheritt, J., and Sandberg, R. D., 2016. "A Novel Evolutionary Algorithm Applied to Algebraic Modifications of the RANS Stress-Strain Relationship". J. Comput. Phys., 325, pp. 22-37.

[28] Weatheritt, J., and Sandberg, R. D., 2017. "The development of algebraic stress models using a novel evolutionary algorithm". Int. J. Heat Fluid Flow, 68(September), pp. 298-318.

[29] Weatheritt, J., Pichler, R., Sandberg, R. D., Laskowski, G., and Michelassi, V., 2017. "Machine Learning for Turbulence Model Development Using a High Fidelity HPT Cascade Simulation". In ASME Turbo Expo 2017 Turbomach. Tech. Conf. Expo., pp. GT2017-63497.

[30] Akolekar, H. D., Weatheritt, J., Hutchins, N., Sandberg, R. D., Laskowski, G., and Michelassi, V., 2019. "Development and Use of Machine-Learnt Algebraic Reynolds Stress Models for Enhanced Prediction of Wake Mixing in Low Pressure Turbines". J. Turbomach., 141(4), p. 041010.

[31] Sandberg, R. D., Tan, R., Weatheritt, J., Ooi, A., Haghiri, A., Michelassi, V., and Laskowski, G., 2018. "Applying Machine Learnt Explicit Algebraic Stress and Scalar Flux Models To a Fundamental Trailing Edge Slot”. J. Turbomach., 140(10), p. 101008.

[32] Rodi, W., 1976. "A New Algebraic Relation for Calculating the Reynolds Stresses”. In Gesellschaft Angew. Math. und Mech. Work. Paris Fr., Vol. 56.

[33] Stadtmüller, P., and Fottner, L., 2001. "A Test Case for the Numerical Investigation of Wake Passing Effects on a Highly Loaded LP Turbine Cascade Blade". ASME Turbo Expo, pp. GT-2001-0311.

[34] Lopez, M., and Walters, D. K., 2016. "Prediction of Transitional and Fully Turbulent Flow Using an Alternative to the Laminar Kinetic Energy Approach". J. Turbul., 17(3), pp. 253-273.

[35] Parneix, S., Laurence, D., and Durbin, P. A., 1998. "A Procedure for Using DNS Databases”. J. Fluids Eng. Trans. ASME, 120(1), pp. 40-46.

[36] Lumley, J. L., 1970. "Toward a turbulent constitutive relation". J. Fluid Mech., 41(2), pp. 413-434.

[37] Michelassi, V., and Wissink, J. G., 2015. “Turbulent Kinetic Energy Production in the Vane of a Low-Pressure Linear Turbine Cascade with Incoming Wakes". Int. J. Rotating Mach., 2015, p. 650783.

[38] Hatman, A., and Wang, T., 1998. "Separated-Flow Transition: Part I Experimental Methodology and Mode Classification”. In Int. Gas Turbine Aeroengine Congr. Exhib. Stock. Sweden.

[39] Hodson, H. P., and Howell, R. J., 2005. "Bladerow Interactions, Transition, and High-Lift Aerofoils in Low-Pressure Turbines". Annu. Rev. Fluid Mech., 37(1), pp. 71-98.

[40] Cumpsty, N. a., and Horlock, J. H., 2006. “Averaging Nonuniform Flow for a Purpose". J. Turbomach., 128(1), p. 120.

[41] Leschziner, M., 2015. Statistical Turbulence Modelling for Fluid Dynamics - Demystified: an Introductory Text for Graduate Engineering Students. World Scientific, Oxford, UK. 


\section{University Library}

\section{- M M N E R VA A gateway to Melbourne's research publications}

Minerva Access is the Institutional Repository of The University of Melbourne

Author/s:

Akolekar, HD;Sandberg, RD;Hutchins, N;Michelassi, V;Laskowski, G

Title:

Machine-Learnt Turbulence Closures for Low-Pressure Turbines With Unsteady Inflow Conditions

Date:

2019-10-01

Citation:

Akolekar, H. D., Sandberg, R. D., Hutchins, N., Michelassi, V. \& Laskowski, G. (2019). Machine-Learnt Turbulence Closures for Low-Pressure Turbines With Unsteady Inflow Conditions. JOURNAL OF TURBOMACHINERY-TRANSACTIONS OF THE ASME, 141 (10), https://doi.org/10.1115/1.4043907.

Persistent Link:

http://hdl.handle.net/11343/241881 